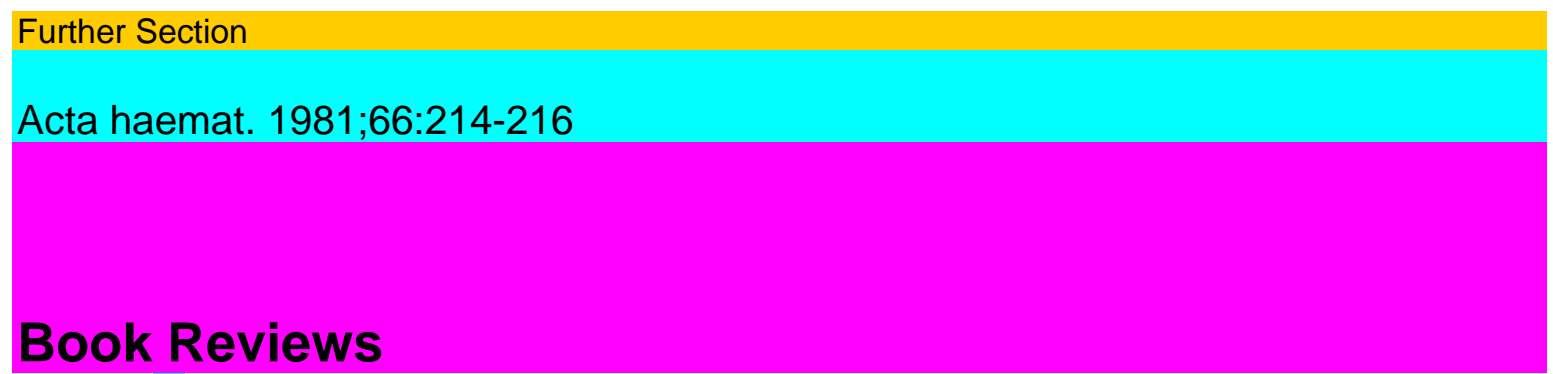

\title{
J.G. Shamo†f
}

Prevention of Venous Thrombosis and

Pulmonary Embolism

MTP Press, Lancaster 1980135 pp.; £8.95 ISBN 0-85200-295-5

The 13 chapters of this monograph cover much more than just the prevention of thromboembolism; each one incorporates the author's suggestion that: 'All hospitalized patients with their limited activity of confinement and/or mobilization may receive small subcutaneous injections of heparin as protection against deep venous thrombosis and pulmonary thromboembolism, just as it is routine for patients to have blood counts... etc' Four chapters are devoted to this small-dose heparin regimen, which implies tight laboratory control of blood coagulation in contradistinction with Kakkar's low-dose heparin regimen. Although the author's concept is basically similar to that proposed by Kakkar, it is more logical because it fulfills essential criteria in that it takes into account not only the patient's weight but also the well-known, often considerable differences between individuals in heparin metabolism. The dosage range lies between 1,500 and 5,000 units of heparin given subcutaneously every $6 \mathrm{~h}$. Daily, before a next injection, the whole-blood clotting time is determined by means of a well-standardized simple and rapid bedside method. By dosage adjustment, the clotting time is kept within the normal limits of IV2-2V2 min. In the author's experience covering 6,000 patients, this regimen gives good clinical results and no untoward bleeding complications.

Unfortunately, the chapters dealing with other aspects contain many dubious statements, misquotations, and references which are ill-related to the text. Important European publications are not referred to, and titles of foreign-language publications are annoyingly misspelled.

It would be interesting to know whether Dr. Sharnoff's recommendation of his regimen for therapy is really useful, especially in view of the negative results obtained with Dr. Kakkar's low-

dose regimen. Also controversial is Dr. Sharnoff's repeated statement that this standardized method of determination of blood clottability: '... measures the action of the oral anticoagulants such as coumadin and warfarin (the former is the trade name, the latter the generic name: EAL) long before the prothrombin time values are altered'.

Those who are interested in individual applica tion of small-dose heparin and anyone not famil iar with Dr. Sharnoff's views presented in his original articles will find both treated exhaustively in the present monograph. E.A. Loeliger H. Schmid-Schönbein, P. Teitel (eds.) Basic Aspects of Blood Trauma Nijhoff, The Hague 1979 IX + 404 pp.; Dfl 120-ISBN 90-247-2279-9 
This book is the result of an international workshop on 'blood trauma' related to artificial organs, especially extracorporeal oxygenators. Discussion of problems associated with 'blood trauma' as induced by artificial organs in clinical and experimental conditions was made possible by the interdisciplinary character of this symposium. Eighteen papers with references to original literature are presented. They mainly concern alterations of thrombocytes, erythrocytes, granulocytes and plasma proteins. Thrombocyte and red cell alterations are discussed with special reference to prehemolytic stages of erythrocytes and platelet activation as induced by physical factors, particularly shear forces. The chapters on the physical chemistry of surface interactions, which can influence the configuration and the functional behaviour of plasma proteins, emphasize the complexity of the problems to be solved. In particular, the pathophysiological consequences of protein alterations are still far from being understood.

The open and stimulating discussions held after each presentation of a paper have been recorded after extensive editorial work and are a very attractive aspect of the book. Some of the methods used for investigation of 'blood trauma' such

Book Reviews

215

as volume determination of thrombocytes and red cells in automatic particle analyzers are called into question. The electro-optical determination of fíbrinogen-fibrin conversion on artificial surfaces is subjected to critical and clarifying remarks by the participants of the symposium.

Focusing on the general biological significance of 'blood trauma' and summarizing the various discussion remarks, H. Schmid-Schönbein underlines the important role of the socalled unspecific defense system of normal human blood which is activated in patients subjected to artificial oxygen-ators.

This book provides excellent and important in

formation for all those who are working in the

clinical, technical or experimental field of artifi

cial organs. M. Stäubli

A.L. de Week (ed.)

Differentiated Lymphocyte Functions

Progress in Allergy, vol. 28 Karger, Basel 1981 X + 286 pp.; SFr. 145.-ISBN 3-8055-1834-X

This latest volume of Progress in Allergy signals an important (and welcome) change in editorial policy: breaking with an over 40-year-old tradition, the present and future issues will contain reviews on closely related topics, thus offering the reader a general overview instead of a series of articles on widely diverse subjects.

The present series opens with a fascinating article by $\mathrm{Oh}<$ no et al. on the evolution of the immune system. The mechanisms by which $\mathrm{V}$ and $\mathrm{C}$ gene coding sequences may fuse are offered as the basis of 'Promethean Foresight' of the immune apparatus, i.e., the ability to react in an epitope-specific fashion against every conceivable molecule. On the other hand, the 'Curse of Prometheus' is viewed to lie in the fact that V gene products unavoidably are also directed against autoantigens, a situation which is held in precarious balance by the evolution of helper and suppressor cells. In the second article, Hämmerling presents a view of B-cell differentiation as derived from induction of phenotypic changes in vitro. Three levels 
of differentiation are considered: (1) development as governed by the general laws of tissue differentiation; (2) diversification with regard to antibody specificity (in part superimposed on the first step); and (3) optional change of the class of immuno-globulin synthesized while maintaining specificity. Roder et al. review present knowledge on 'natural killer' (NK) cells (300 references!). Evidence suggests that these cells constitute a rather heterogen-ous population and that they might play an important role in resistance to the growth of certain tumors and, possibly, in the elimination of virus-infected cells. Among the various cell-mediated cy-totoxic mechanisms, those of NK cells appear unique since they do not seem to be under $\mathrm{H}-2$ restriction, lack classical memory, and act upon a wide range of unrelated cells with some degree of specificity. The article by Battisto and Ponzio addresses itself to autologous and syngeneic mixed lymphocyte reactions (MLR) in mouse and man. Because the replicating cell is always a T cell -stimulated by B cells at different differentiation stages, macrophages and dendritic cells -, studies of MLR may yield information on the function of subsets of thymus-derived cells. While in mice two types of 'isogenic lymphoyte interaction' may be distinguished on the basis of both stimulator and responder cells, only one counterpart, the so-called autologous MLR, has been described in man. During this anti-self reaction, cytotoxic cells fail to be generated; however, soluble helper factors for precursors of cytotoxic T cells (TRF?) may be produced, and suppressor activity may be found in supernatants. The significance of these phenomena in vivo is still unknown. In the last review. Parrott and Wilkinson focus attention on lymphocyte locomotion as an active process and try to relate observations on the migratory behavior of lymphocytes in vivo to patterns of locomotion as studied in vitro. Since recirculating lymphocytes have to 'pass through many capillary beds' of lymphoid and non-lymphoid organs, interactions of these cells with endothelia of blood vessels - together with mechanisms regulating blood flow - strongly influence lymphocyte distribution. However, exactly why certain lymphocytes accumulate in certain organs or sites remains poorly understood, and, at present, observations in vitro offer no easy explanation for mechanisms governing migratory behavior of lymphocytes in vivo.

Lecture of this volume is highly recommended for immunologists and biologists who look for a

216

Book Reviews

'tour d'horizon' of selective topics of lymphocyte differentiation.M.W. Hess, Bern

B.A. Brown

Hematology: Principles and Procedures

Lea \& Febiger, Philadelphia 1980 IX + 358 pp.; US\$ 22.00 ISBN 0-8121-0707-1

In this new edition the concept of the textbook remains unchanged. In several chapters additions have been made, photographs have been replaced and excellent new color plates have been added. The purpose of the book remains the same: to acquaint the new student with a basic knowledge of hematology. All important methods used in laboratory practice are given in detail, and a chapter is dealing with automation. According to the main purpose of the textbook, the description of diseases is limited to 33 pages. The bibliography contains a useful selection of 155 references. The book is a valuable source of information for medical students and the laboratory staff. 
H.R. Marti, Aarau

W.A. Schroeder, T.H.J. Huisman The Chromatography of Hemoglobin

Clinical and Biochemical Analysis Series,

vol. 9

Marcel Dekker, New York 1980

255 pp.; SFr. 68.-

ISBN 0-8247-6941-4

This book, written by two outstanding experts in hemoglobin biochemistry, is a most valuable source of information for hematologists and biochemists dealing with hemoglobin variants. There are chapters on the basic principles and detailed technical procedures of macrochroma-tography on cation and anion exchangers, micro-chromatography, chromatography of hemoglobin A1C and other minor components, of hemoglobin F and $\gamma \mathrm{t}$, on the preparative chromatography and the chromatography of animal hemoglobins. In an appendix the hemoglobin variants quan-titated or isolated by ion-exchange chromatography are listed. More than 140 references are given. The comprehensive laboratory manual can be recommended to all scientists interested in the biological applications of chromatography.

H.R. Marti, Aarau 